\title{
ARTICLE Prostaglandin E1 attenuates high glucose-induced apoptosis in proximal renal tubular cells by inhibiting the JNK/Bim pathway
}

\author{
Yu-han Zhang ${ }^{1}$, Ya-qin Zhang ${ }^{1,2}$, Cong-cong Guo ${ }^{3,4}$, Li-kang Wang ${ }^{1,3}$, Yu-jiao Cui ${ }^{1,3}$, Jian-jun Dong ${ }^{5}$ and Lin Liao ${ }^{1,6}$
}

Proximal renal tubular damage is a critical process underlying diabetic kidney disease (DKD). Our previous study shows that prostaglandin E1 (PGE1) reduces the apoptosis of renal tubular cells in DKD rats. But its underlying mechanisms remain unclear. In this study we investigated the protective effects of PGE1 in DKD rats and high glucose (HG, $30 \mathrm{mM})$-treated HK-2 proximal tubular cells. Four weeks after uninephrectomized streptozotocin-induced diabetic rats were established, the DKD rats were administered PGE1 $\left(10 \mu \mathrm{g} \cdot \mathrm{kg}^{-1} \cdot \mathrm{d}^{-1}\right.$, iv.) for 10 consecutive days. We showed that PGE1 administration did not change blood glucose levels, but alleviated diabetic kidney injury in the DKD rats, evidenced by markedly reduced proteinuria and renal tubular apoptosis. In the in vitro experiments, PGE1 (0.1-100 $\mathrm{MM}$ ) significantly enhanced HG-reduced HK-2 cell viability. In HG-treated HK-2 cells, PGE1 (10 $\mu \mathrm{M})$ significantly suppressed the c-Jun N-terminal kinase (JNK) and the mitochondrial apoptosis-related protein expressions such as Bim, Bax, caspase-3 and cleaved caspase-3; similar changes were also observed in the kidney of PGE1-treated DKD rats. By using two pharmacological tools-JNK activator anisomycin (AM) and JNK inhibitor SP600125, we revealed that PGE1 blocked HG-triggered activation of JNK/Bim pathway in HK-2 cells; JNK was an upstream regulator of Bim. In conclusion, our results demonstrate that the nephroprotective effects of PGE1 against apoptosis of proximal renal tubule in DKD rats via suppressing JNK-related Bim signaling pathway.

Acta Pharmacologica Sinica (2020) 41:561-571; https://doi.org/10.1038/s41401-019-0314-9

\section{INTRODUCTION}

Diabetic kidney disease (DKD), a major microvascular complication occurring in $20 \%-40 \%$ of patients with diabetes mellitus (DM), is the leading cause of end-stage renal failure worldwide [1]. Various factors contribute to the development of DKD. Hyperglycemiainduced glomerular injury has been recognized as a classic characteristic of the pathogenesis of DKD [2-4]. However, emerging evidence suggests that proximal renal tubular injury also plays a pivotal role in the initial kidney damage associated with DKD, which occurs earlier than glomerulopathy [5-7]. Thus, proximal renal tubular cells may be a new therapeutic target for the treatment of DKD.

Proximal renal tubules are an energy-consuming machines that participate in the reabsorption of fluid and are susceptible to a variety of metabolic and hemodynamic factors [8] that may induce apoptosis. Apoptosis has been found in proximal renal tubules and distal renal tubules in diabetic patients [9]. In addition, DKD animals and proximal renal tubular cells in vitro undergo apoptosis in a high-glucose (HG) environment [10, 11]. From this evidence, we can consider the apoptosis of tubular cells to be a vital feature of DKD [12].
The proapoptotic $\mathrm{Bcl}-2$ homology domain 3-only protein Bim plays an important role in initiating the intrinsic mitochondrial apoptotic pathway under pathophysiological conditions [13]. Many stimuli, such as HG stimulation, can lead to the activation of Bim-induced apoptosis [14, 15]. Our previous study indicated that HG induces apoptosis via the upregulation of proapoptotic Bim expression in HK-2 cells [5]. Bim directly activates Bax/Bak on the mitochondrial outer membrane, and this activation is accompanied by the indirect activation of the antiapoptotic proteins $\mathrm{BCl}-2, \mathrm{Bcl}-\mathrm{xL}$, and $\mathrm{Mcl}-1$. Following the release of cytochrome c, caspase-3, and caspase-9, cell apoptosis is eventually induced [16].

c-Jun N-terminal kinase (JNK) is an important regulator of apoptosis, which can be activated by many stimuli in the external environment, including HG $[17,18]$. The expression of proapoptotic Bim can be transcriptionally induced by JNK, leading to Bimdependent apoptosis [19-21].

Prostaglandin E1 (PGE1) is a 20-carbon unsaturated fatty acid that is found widely in various species of mammals. Exogenous PGE1 is a powerful vasodilator and platelet aggregation inhibitor that has a wide range of pharmacological applications. In recent

\footnotetext{
${ }^{1}$ Division of Endocrinology, Department of Internal Medicine, Shandong Provincial Qianfoshan Hospital, Shandong University, Ji-nan 250014, China; ${ }^{2}$ Division of Endocrinology, Department of Internal Medicine, Wuhan Third Hospital, Wuhan 430060, China; ${ }^{3}$ First Clinical Medical College, Shandong University of Traditional Chinese Medicine, Ji-nan 250355, China; ${ }^{4}$ Department of Endocrinology, Affiliated Hospital of Shandong University of Traditional Chinese Medicine, Ji-nan 250011, China; ${ }^{5}$ Division of Endocrinology,

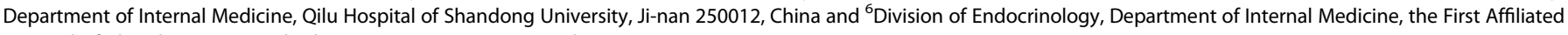
Hospital of Shandong First Medical University, Ji-nan 250014, China
}

Correspondence: Jian-jun Dong (cwc_Il@sdu.edu.cn) or Lin Liao (liaolin@sdu.edu.cn)

These authors contributed equally: Yu-han Zhang, Ya-qin Zhang

Received: 9 May 2019 Accepted: 30 September 2019

Published online: 4 November 2019 
years, PGE1 has been found to reduce apoptosis in other animals and tissues [22-25]. Our previous experiment demonstrated that PGE1 reduces the apoptosis of renal tubular cells in DKD rats [25]. However, it is unclear whether PGE1 can reduce apoptosis by modulating Bim, and it remains to be investigated whether the JNK/Bim signaling pathway is involved.

\section{MATERIALS AND METHODS}

Animal models and treatment

Male Wistar rats (initial mean body weight: $200 \pm 20 \mathrm{~g}$ ) were purchased from Beijing HFK Bio-Technology Co. Ltd. (Beijing, China, Certificate No. SCXK 2014-0008). All animals were kept in a light-controlled room with constant room temperature $\left(21^{\circ} \mathrm{C}\right)$ and humidity (75\%). Water and standard chow were available ad libitum. All rats were randomly divided into four groups: (1) the sham group $(n=6) ;(2)$ the unilaterally nephrectomized group (Unx, $n=6)$; (3) the Unx + streptozotocin (STZ) group (DKD, $n=6$ ); and (4) the Unx + STZ + PGE1 group (PGE1, $n=6$ ). Right nephrectomy was employed to accelerate the development of renal injury [26]. Seven days after uninephrectomy, diabetes was induced by a single intraperitoneal injection of STZ (Sigma-Aldrich, St Louis, MO, USA) at a dose of $45 \mathrm{mg} / \mathrm{kg}$ body weight diluted in citrate buffer $(0.1 \mathrm{~mol} / \mathrm{L}, \mathrm{pH} 4.0)$, as previously described [27]. The presence of diabetes (blood glucose levels over $16.7 \mathrm{mmol} / \mathrm{L}$ ) was confirmed $72 \mathrm{~h}$ after STZ injection by measuring tail blood glucose levels. The blood glucose levels of all rats were measured weekly using a One Touch Ultra blood glucose monitoring system (LifeScan Inc., Milpitas, CA, USA). Each sham control rat received sham operation without damage to the kidney, as previously described [26]. To induce DKD, the rats were left in a diabetic condition without any treatment for the following 4 weeks [28]. The DKD model was considered to be successfully established in animals that produced over 1.5 times more urine than they produced before the procedure and that exhibited 24-h microalbuminuria levels that were 10 times higher than those in the control group [25]. The rats in the sham and Unx groups that did not receive STZ injection were used as nondiabetic controls. Meanwhile, hematoxylin and eosin (H\&E), periodic acid-Schiff (PAS) and periodic acid silver methenamine (PASM) staining was performed in random kidney samples to verify the establishment of the DKD model. In the PGE1-treated group, PGE1 (Lipo-PGE1, Beijing Taide Pharmaceutical Company, Beijing, China) at a dose of $10 \mu \mathrm{g} / \mathrm{kg}$ body weight was injected into the tail vein daily for 10 consecutive days [29], while diabetic animals that received vehicle control without PGE1 were used as the negative treatment control (DKD group). After $10 \mathrm{~d}$ of PGE1 treatment, the left kidney was then harvested for morphological studies and molecular analyses. The study was approved by the Institutional Animal Care and Use Committee of Shandong Provincial Qianfoshan Hospital and was performed in accordance with the Principles of Laboratory Animal Care.

\section{Assay of albuminuria}

Urinary protein levels were assessed by the Bradford method. The levels of urinary albumin were determined with an ELISA kit (Cusabio Biotech Co., Wuhan, China) according to the manufacturer's instructions.

\section{Renal histology}

The removed kidneys were fixed in $4 \%$ phosphate-buffered formalin solution and embedded in paraffin. Three-micrometerthick sections were stained with H\&E, PAS, and PASM. The morphology and structure of the kidney tissue were visualized by H\&E staining. PAS staining was used to demonstrate mesangial matrix deposition, and basement membrane thickening was assessed by PASM staining. The degree of glomerular damage was defined as the extent of extracellular matrix deposition and mesangial expansion [30]. Tubulointerstitial changes included tubular atrophy, casts, tubular dilation, interstitial mononuclear cell, and extracellular matrix accumulation [31]. All renal biopsies were examined by the same pathologist, who was blinded to the experimental groups.

Immunohistochemical analysis

Three-micrometer-thick paraffin sections were used to evaluate $\mathrm{Bim}, \mathrm{Bax}$, and $\mathrm{Bcl}-2$ expression using a high pressure-based antigen retrieval technique. The following primary antibodies were used: rabbit anti-Bim (1:100, Abcam, Cambridge, MA, USA); rabbit anti-Bax (1:100, Affinity, Chicago, IL, USA); rabbit anti-Bcl-2 (1:100, Affinity). These antibodies were added to the sections and incubated overnight at $4{ }^{\circ} \mathrm{C}$. Negative controls were incubated with PBS. After washing with PBS, the sections were incubated with a horseradish peroxidase-labeled goat anti-rabbit IgG secondary antibody (Beijing Zhongshan Golden Bridge Biotechnology Co. Ltd., Beijing, China) at room temperature for $1 \mathrm{~h}$, followed by color development with diaminobenzidine (DAB) and counterstaining with hematoxylin. The sections were examined under a light microscope (Olympus, Tokyo, Japan).

\section{Cell culture}

The human proximal renal tubular cell line HK-2 (American Type Cell Collection, Rockville, MD, USA) was obtained from Wuhan Pricells Biotechnology \& Medicine Co. (Wuhan, China) and cultured in RPMI-1640 medium supplemented with $11.1 \mathrm{mM} \mathrm{D-}$ glucose, $10 \%$ fetal bovine serum and $1 \%$ penicillin-streptomycin (HyClone, Logan, UT, USA) in an incubator with $5 \% \mathrm{CO}_{2}$ at $37^{\circ} \mathrm{C}$. For most trials, the cells were incubated in medium containing 5.5 $\mathrm{mM}$ D-glucose + $24.5 \mathrm{mM}$ D-mannitol [as a medium-glucose (MG) control group] or $30 \mathrm{mM} \mathrm{D-glucose} \mathrm{(HG} \mathrm{group).} \mathrm{D-mannitol} \mathrm{and} \mathrm{D-}$ glucose were purchased from Sigma-Aldrich Canada Ltd. (Oakville, ON, Canada). Cells were cultured in the presence or absence of $10 \mu \mathrm{M}$ PGE1 (National Institute for Food and Drug Control, Beijing, China), $0.2 \mu \mathrm{M}$ anisomycin (a JNK agonist) (MedChemExpress, Monmouth Junction, NJ, USA) and $10 \mu \mathrm{M}[32$, 33] SP600125 (a JNK inhibitor) (MedChemExpress) for $48 \mathrm{~h}$.

\section{Cell counting kit-8 assay}

HK- 2 cells were cultivated in 96-well plates at a density of $3.0 \times 10^{4}$ cells $/ \mathrm{cm}^{2}$ in RPMI-1640 medium overnight. The cells were treated with different concentrations of PGE1 $(0.1,1,10$, and $100 \mu \mathrm{M})$ in HG solution (30 mM D-glucose). After 24,48 , and $72 \mathrm{~h}, 10 \mu \mathrm{L}$ of Counting Kit-8 reagent (Dojindo Molecular Technologies, Inc., Kumamoto, Japan) was added to each well. The culture plates were incubated for an additional $2 \mathrm{~h}$ in a humidified $\mathrm{CO}_{2}$ incubator at $37^{\circ} \mathrm{C}$. Finally, the absorbance of each well at $450 \mathrm{~nm}$ was measured by a microplate reader (Thermo Fisher Scientific, Inc., Waltham, MA, USA). Five wells were prepared for each concentration, and the experiment was repeated 3 times. The cell survival rate was calculated according to the manufacturers' instructions.

Flow cytometry analysis of apoptosis

Analysis of the apoptotic effects of PGE1 on HK-2 cells was performed using the Annexin V-FITC kit (Neobioscience, Inc., Shenzhen, China). Following the recommended protocols, cells were cultured in 6-well plates overnight and then exposed to 5.5 $\mathrm{mM}$ glucose $+24.5 \mathrm{mM}$ mannitol, $30 \mathrm{mM} \mathrm{HG}$, and HG + PGE1 for the indicated times. After $48 \mathrm{~h}$, the cells were detached with trypsin-EDTA, washed twice with ice-cold PBS, collected by centrifugation, and resuspended in $500 \mu \mathrm{L}$ of $1 \times$ binding buffer. Thereafter, a total of $5 \mu \mathrm{L}$ of annexin V-FITC and $10 \mu \mathrm{L}$ of propidium iodide $(\mathrm{PI})$ in binding buffer were added and incubated for $30 \mathrm{~min}$ at room temperature in the dark. The apoptosis rates of the cell samples were analyzed with a flow cytometer (BD FACSAria $^{\mathrm{TM}}$ II Cell Sorter, Franklin Lakes, NJ, USA). Cells positive for annexin V-FITC or negative for PI were considered apoptotic cells. 
For each sample, 10000 events were acquired. Analysis was carried out following at least three separate experiments.

\section{Immunofluorescent staining}

Three-micrometer-thick frozen kidney sections were prepared. HK-2 cells were seeded on slides in a six-well plate and stimulated with different media for $48 \mathrm{~h}$. The sections and slides were fixed for 10 min with $4 \%$ paraformaldehyde and then permeabilized with $0.05 \%$ Triton $\mathrm{X}-100$ for $15 \mathrm{~min}$. Then, the sections and slides were incubated with a rabbit anti-Bim antibody $\left(1: 150\right.$; Abcam) at $4{ }^{\circ} \mathrm{C}$ overnight. After incubation with Alexa Fluor 546-conjugated donkey anti-rabbit $\lg G(H+L)(1: 200$, Invitrogen, CA, USA) for $1 \mathrm{~h}$ at room temperature in the dark, the sections and slides were stained with diphenyleneiodonium (DAPI). Images were detected using a fluorescence microscope (Olympus FSX100). The intensity of fluorescence indicated the expression level of the Bim protein.

\section{TUNEL assay}

The apoptosis of renal tubules and glomeruli was detected in situ in 7- $\mu$ m-thick frozen kidney tissue sections, as well as in HK-2 cells on slides after different treatments. The TUNEL assay was performed using an In Situ Cell Death Detection kit (Roche, Basel, Switzerland). The apoptosis rate of kidney tissues and HK-2 cells were calculated according to the manufacturer's instructions.

Western blot analysis

Renal cortical tissues were lysed with radioimmunoprecipitation assay (RIPA) buffer (150 mM sodium chloride, 1\% Triton X-100, 1\% sodium deoxycholate, $0.1 \%$ sodium dodecyl sulfate, $50 \mathrm{mM}$ Tris, $\mathrm{pH} 7.4$, sodium orthovanadate, sodium fluoride, EDTA, and leupeptin) with protease and phosphatase inhibitors (Beyotime, Inc., Shanghai, China) to extract proteins as described previously [34]. The cells were harvested and lysed in RIPA buffer as described above. Primary antibodies against Bim (1:500, Abcam), Bax (1:1000, Affinity), caspase-3 (1:500, Abcam) cleaved caspase-3 (1:500, Chemicon, Chicago, IL, USA), total JNK (1:500, Affinity), phosphorylated JNK (1:500, Affinity), and $\beta$-actin (ZSBIO, Beijing, China) and conjugated secondary antibodies (goat anti-rabbit lgG) were used. Signals were detected with the Alpha chemiluminescent gel imaging system FluorChem E (ProteinSimple, San Jose, CA, USA) and quantitated with ImageJ software (National Institutes of Health, Bethesda, MD, USA). The expression levels of the examined proteins were normalized to those of $\beta$-actin.

\section{Quantitative RT-PCR}

Total RNA was isolated from kidney tissues and cultured HK-2 cells by TRIzol reagent (Invitrogen, CA, USA). Reverse transcription was carried out by a Prime-Script RT reagent kit (TaKaRa, Dalian, China). Quantitative RT-PCR (qRT-PCR) was optimized and performed using SYBR Green master mix (Vazyme, Nanjing, China) according to the manufacturer's protocol. Fold induction was calculated using the $2^{-\Delta \Delta \mathrm{Ct}}$ method. GAPDH and $\beta$-actin served as the endogenous controls. The primers that were used are shown in Table 1.

\section{Statistical analysis}

The data are expressed as the means \pm SEM. Student's $t$-test was used to assess the significance of the data between the two groups. Comparisons of the variables were performed by one-way ANOVA followed by post hoc analysis using the least significant difference method. A $P<0.05$ was considered to indicate statistical significance.

\section{RESULTS}

Animal experiments and the establishment of the DKD model The protocol for the animal assays is presented in Fig. 1a. Day 0 was the point before operation (sham and Unx). Day 10 was $7 \mathrm{~d}$ after operation and $72 \mathrm{~h}$ after STZ injection, which was also the point at which the diabetes model was established. Day 38 was the point at which the DKD model was established. It also marked the beginning of PGE1 injections. Day 48 was when daily PGE1 intervention ended and all rats were sacrificed. Some diabetic rats and nondiabetic rats were sacrificed to confirm the establishment of the DKD model. The establishment of the DKD model was verified through pathological diagnosis by $\mathrm{H} \& \mathrm{E}, \mathrm{PAS}$, and PASM staining (Fig. 1b). The degrees of glomerular sclerosis and glomerular capillary occlusion were significantly greater in the diabetic groups than in the nondiabetic groups, as indicated by $\mathrm{H} \& \mathrm{E}$ staining. The thickening of the glomerular mesangial matrix, as observed by PAS staining, and the thickening of the glomerular and tubular basement membranes, as observed by PASM staining, were observed to be more severe in the diabetic groups than in the nondiabetic groups. These results indicated that the DKD model was established successfully.

PGE1 had no effect on blood glucose but reduced urinary microalbumin levels in DKD rats

Blood glucose was detected on days $0,10,38$, and 48 . The blood glucose of the diabetic rats was significantly higher than that of the nondiabetic rats. However, there was no significant difference in blood glucose levels after PGE1 intervention compared with those in the DKD rats (Fig. 1c). We next explored the effect of PGE1 on alleviating urinary protein. Twenty-four-hour urine samples were collected on days 0,38 , and 48 . There was an elevated level of microalbuminuria in the DKD group compared with that in the sham and Unx groups on day $38(P<0.05$, Fig. $1 \mathrm{~d})$, in accordance with previous findings in the DKD model [25]. Additionally, the level of microalbuminuria in the PGE1 group decreased compared with that in the DKD group after $10 \mathrm{~d}$ of PGE1 treatment, and the difference was statistically significant $(P<0.05$, Fig. $1 \mathrm{~d})$.

PGE1 reduced the apoptosis of tubular cells in DKD rats To evaluate the apoptosis of the renal cells, a TUNEL assay was conducted for the four groups (the sham, Unx, DKD, and PGE1 group). Apoptotic cells exhibited blue-green fluorescence after the images were merged. Surprisingly, there was almost no apoptosis in the glomeruli. However, apoptosis occurred mainly in the proximal renal tubules and distal renal tubules, as indicated by the red arrows (Fig. 2a). Notably, the apoptosis rate was $11.56 \% \pm$ $0.49 \%$ in the DKD group, which was higher than that in the control groups $(0.85 \% \pm 0.08 \%)(P<0.01$, Fig. $2 b)$. An improvement in apoptosis was clearly observed in the PGE1 group $(3.33 \% \pm 0.11 \%)$ compared with the DKD group $(P<0.01$, Fig. $2 b)$.

PGE1 attenuated Bim, Bax, caspase-3, and cleaved caspase-3 expression in DKD rats

To determine whether apoptosis-related proteins participate in the attenuation of apoptosis by PGE1, we evaluated the expression of the apoptosis protein Bim in the kidneys of rats. Bim expression in rat kidneys was evaluated by immunofluorescence staining. The red fluorescence intensity indicated how much Bim was expressed. The staining was strongest in the DKD group. After $10 \mathrm{~d}$ of treatment with PGE1, the Bim fluorescence intensity was attenuated compared with that in the DKD group (Fig. 2c). Next, we evaluated the expression of apoptosis-related proteins by Western blotting and qRT-PCR. The results indicated that the protein and gene expression of Bim, Bax, caspase- 3 and cleaved caspase- 3 was increased in the DKD group compared with that in the sham and Unx groups. Conversely, PGE1 reduced the expression of these apoptosis-related proteins compared with that in the DKD group (Fig. 2d-f).

PGE1 alleviated histomorphological injury in DKD rats Histomorphological changes were observed in tissues stained with PAS after PGE1 treatment (Fig. 3). The morphological PAS staining 
Table 1. Primer sequences

\begin{tabular}{lll}
\hline Gene name & Forward-sequence (5'-3') & Reverse-sequence (5'-3') \\
\hline Bim (human) & ATTACCAAGCAGCCGAAGAC & TCCGCAAAGAACCTGTCAAT \\
Bax (human) & AAGCTGAGCGAGTGTCTCCGGCG & CAGATGCCGGTTCAGGTACTCAGTC \\
Caspase-3 (human) & AAGCTGAGCGAGTGTCTCCGGCG & CAGATGCCGGTTCAGGTACTCAGTC \\
Bim (rat) & ATCTTCTCTGCTGTCCCGGTC & TGCCTTATGGAAGCCATTGCAC \\
Bax (rat) & GAATTGGCGATGAACTGGAC & GCAAAGTAGAAAAGGCAACC \\
Caspase-3 (rat) & GACTGCGGTATTGAGACAGA & CGAGTGAGGATGTGCATGAA \\
\hline
\end{tabular}

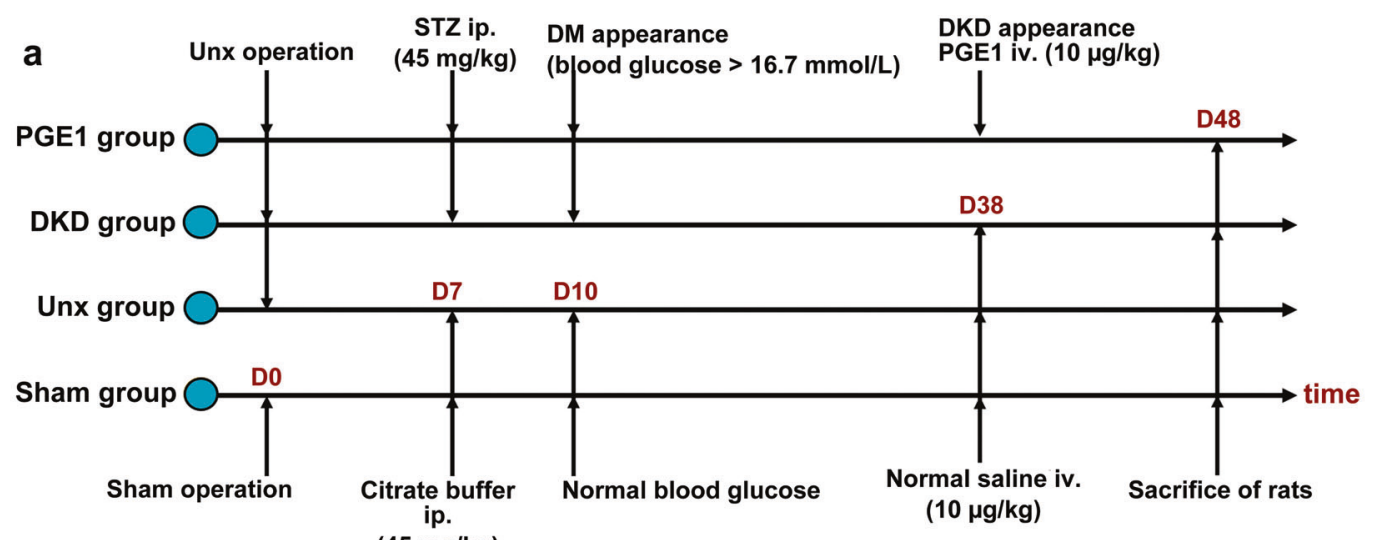

b

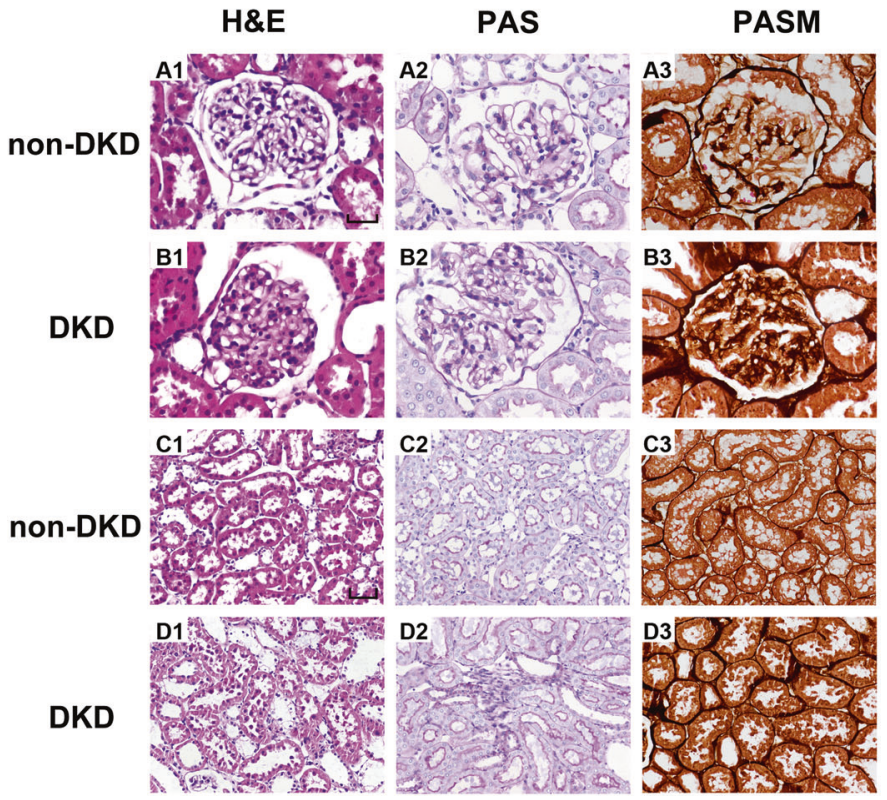

C

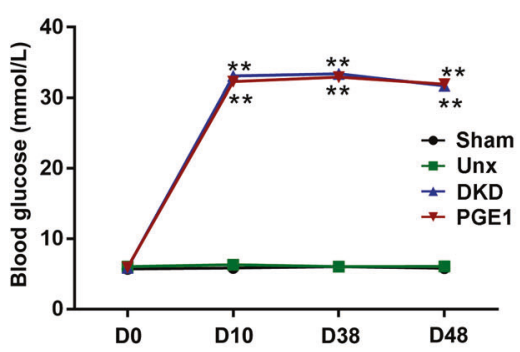

d

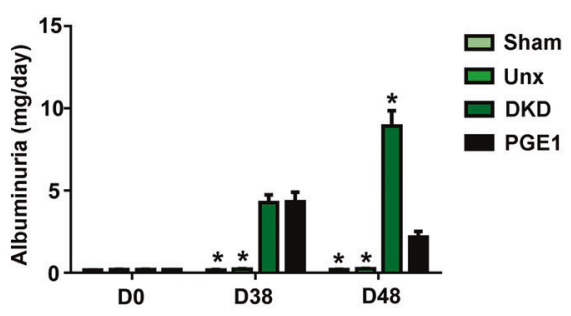

Fig. 1 PGE1 had no effect on blood glucose but reduced urinary microalbumin levels after the establishment of the DKD model. a General flow chart of the animal experiments. Each experimental group contained six rats, and timelines of DKD model establishment and STZ and PGE1 injections are displayed. The injection volumes for STZ and PGE1 in the rats were $45 \mathrm{mg} / \mathrm{kg}$ and $10 \mu \mathrm{g} / \mathrm{kg}$, respectively. b H\&E, PAS and PASM staining were used to confirm the establishment of the DKD model. A1-B3 mainly show glomeruli ( $\times 400$ magnification). Scale bar, 30 $\mu \mathrm{m}$. C1-D3 show renal tubules ( $\times 200$ magnification). Scale bar, $50 \mu \mathrm{m}$. Non-DKD kidneys were from the sham and Unx groups. c Changes in the blood glucose levels $(\mathrm{mmol} / \mathrm{L})$ of the different groups on day 0 , day 10 , day 38 , and day 48 . The data represent the means \pm SEM $(n=6$ per group). ${ }^{* *} P<0.01$ versus the Unx group. $\mathbf{d}$ Twenty-four-hour urinary protein levels were measured by an ELISA kit on day 0 , day 38 , and day 48 . The data represent the means \pm SEM ( $n=6$ per group). ${ }^{*} P<0.05$ versus the PGE1 group 

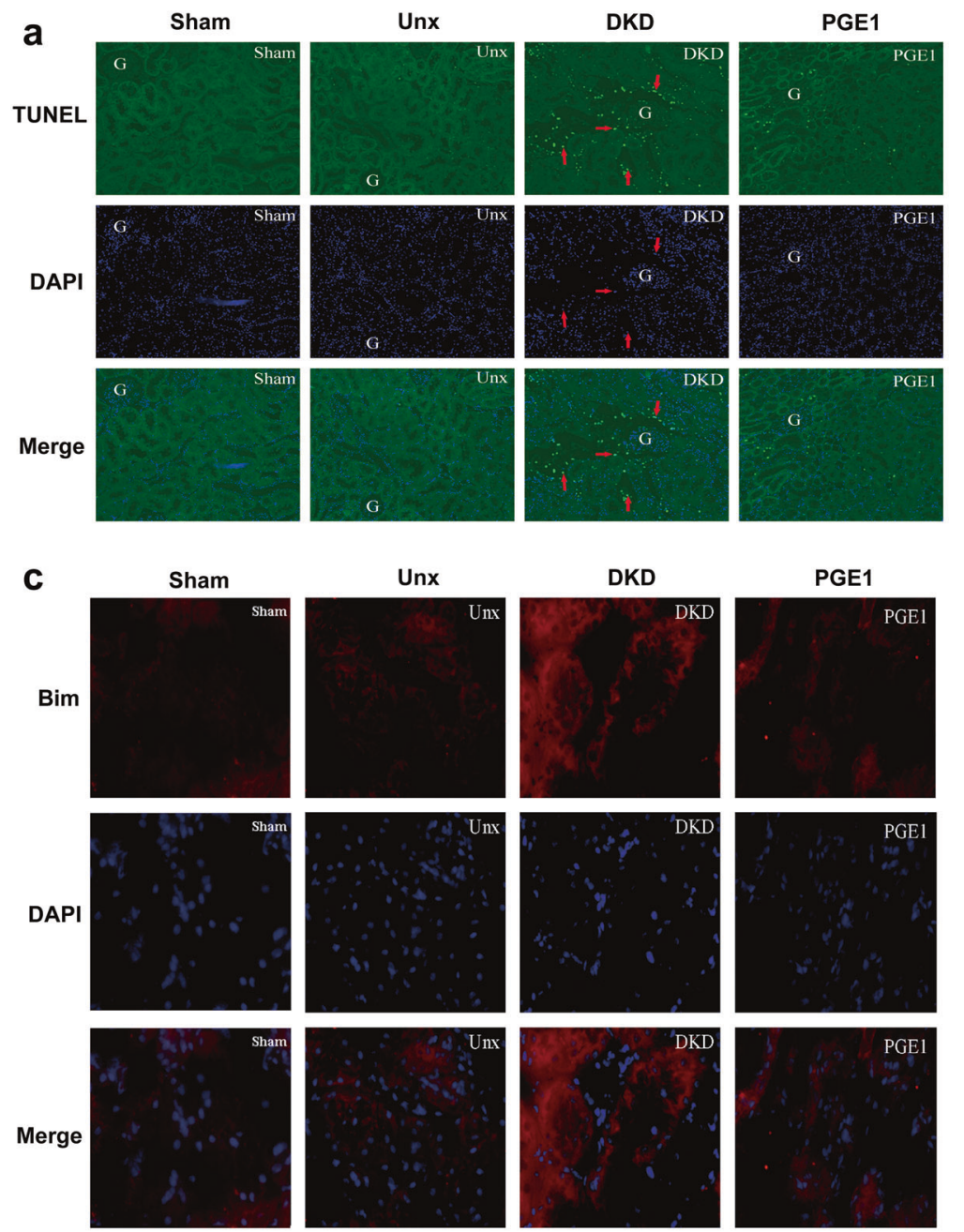

b

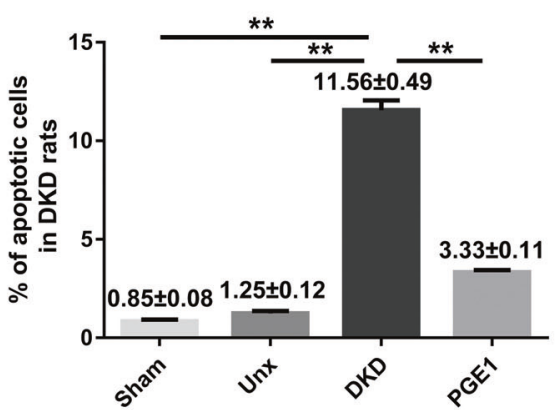

d

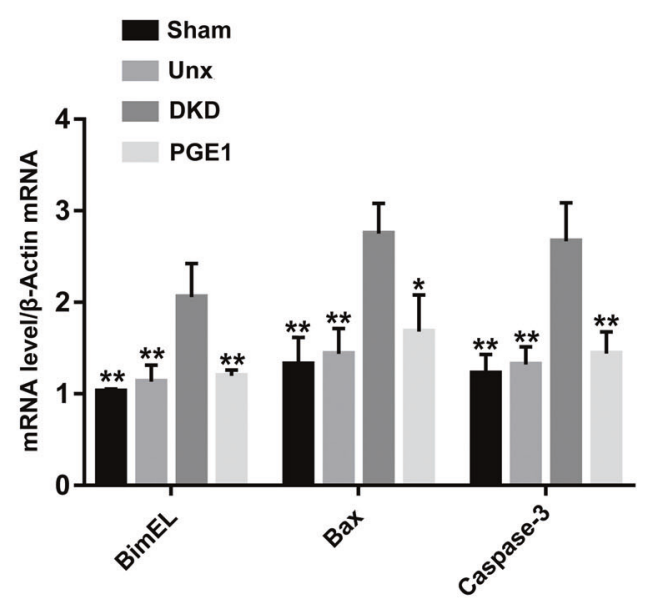

e

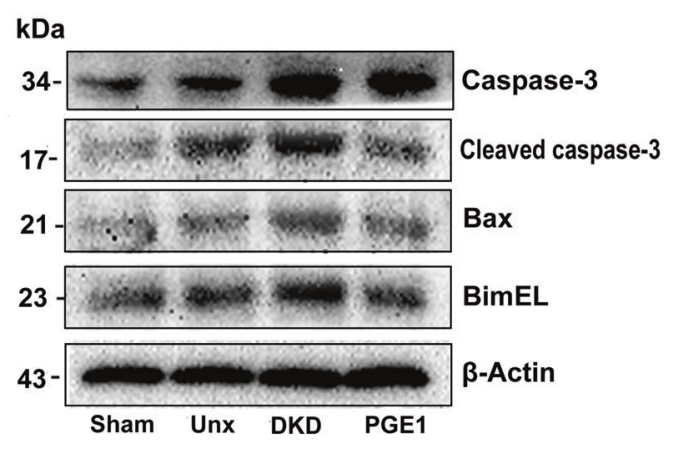

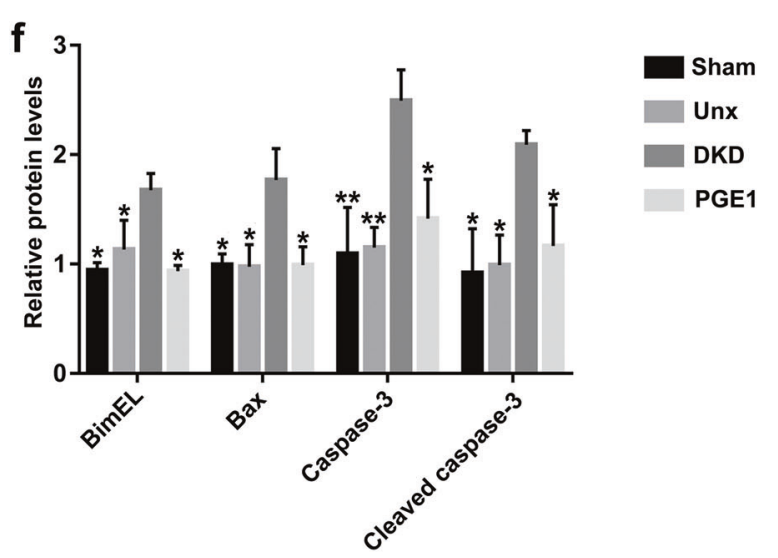

Fig. 2 PGE1 reduced the apoptosis of tubular cells and attenuated Bim, Bax, caspase-3 and cleaved caspase-3 expression in DKD rats. a The apoptosis of tubular cells in rat kidneys was assessed by TUNEL staining ( $\times 200$ magnification). The green fluorescence indicates apoptotic cells, the blue fluorescence indicates nuclei, and the red arrows show apoptotic cells. $\mathbf{b}$ Quantitative analysis of the apoptosis of renal tubular cells. The number of apoptotic cells and the total number of cells in 100 renal tubule regions and the number of apoptotic cells in 50 glomerular regions were determined in a continuous field of vision. The apoptosis rate of renal tubular cells was calculated based on the following formula: the number of apoptotic cells in every 100 renal tubule regions/ the number of total cells $\times 100 \%$. The rate of apoptosis of the glomeruli was determined by calculating the average number of apoptotic cells in each single glomerular region. c Bim expression was detected by immunofluorescence ( $\times 200$ magnification). The red fluorescence represents Bim expression, and the blue fluorescence represents nuclei. d qRT-PCR was performed to determine the mRNA levels of Bim, Bax and caspase-3 in the renal cortex of the rats. $\mathbf{e}, \mathbf{f}$ The protein levels of Bim, Bax, caspase-3 and cleaved caspase- 3 in the renal cortex of the rats were assessed by Western blotting. The data represent the means \pm SEM ( $n=6$ per group). ${ }^{*} P<0.05,{ }^{* *} P<0.01$ versus the DKD group. G glomeruli 

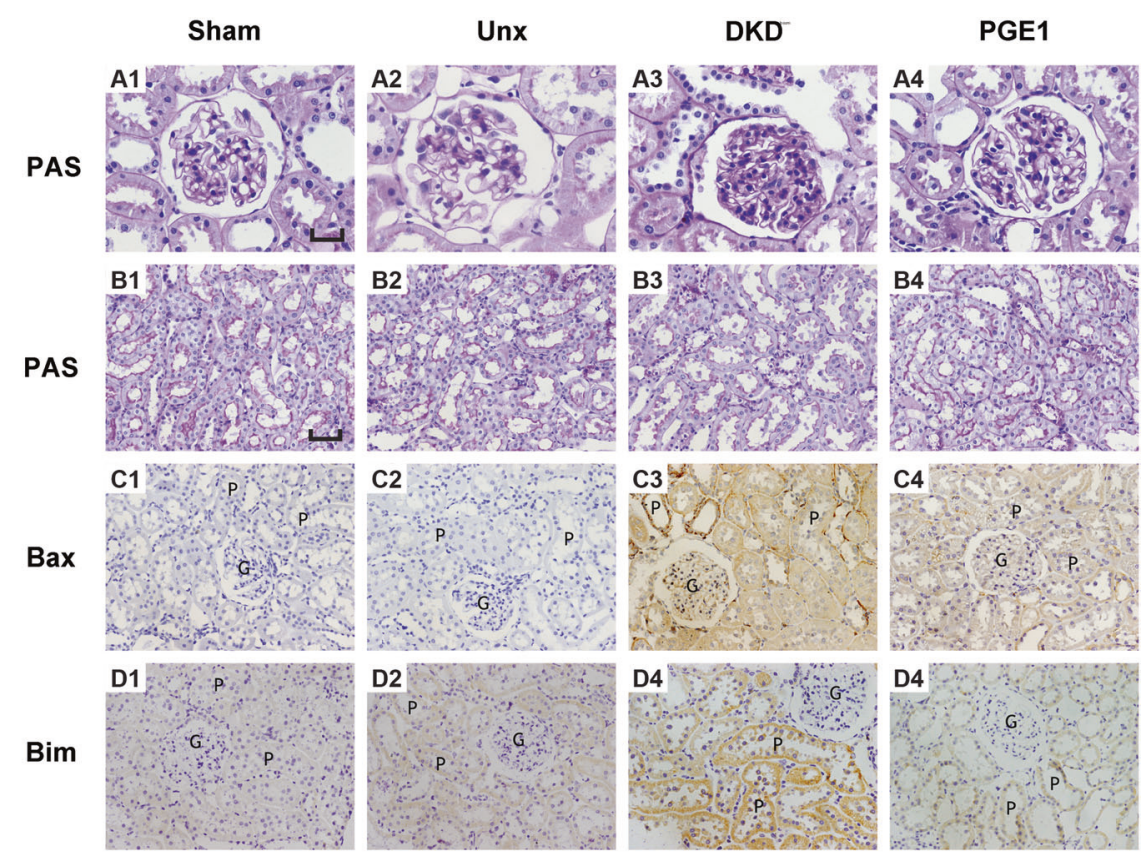

Bcl-2
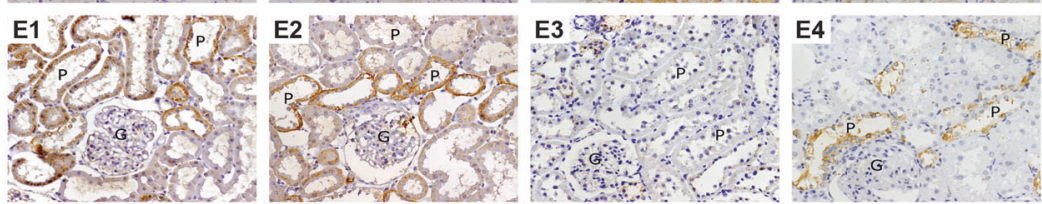

Fig. 3 PGE1 alleviated histomorphological injury in DKD rats. A1-E4: PAS and immunohistochemical staining of the renal cortex were used to observe the effect of PGE1 on histomorphological changes in DKD kidneys at the end of the experimental period. A1-A4: $\times 400$ magnification. Scale bar, $30 \mu \mathrm{m}$. B1-E4: $\times 200$ magnification. Scale bar, $50 \mu \mathrm{m}$. A1-B4: PAS staining of glomeruli and renal tubules on day 48. C1-E4: Immunohistochemical staining for Bax, Bim and $\mathrm{Bcl}-2$ on day 48. G glomeruli, P proximal tubule; the yellow and dark yellow areas represent positively stained regions

of the kidney demonstrated marked alleviation of the thickening of the glomerular basement membrane and an expansion of the mesangium in DKD rats after PGE1 treatment. Renal tubular epithelial cells were detached, and the lumen was enlarged in the DKD group. Renal tubular lesions were observed to be improved in the PGE1 group. Moreover, the effects of PGE1 on renal morphology were reflected by immunohistochemistry. The expression of Bim and Bax was reduced after PGE1 treatment, as determined by immunohistochemistry, while the expression of the antiapoptotic protein $\mathrm{Bcl}-2$ was elevated in the treatment group.

PGE1 increased the cell viability and reduced the apoptosis of HK2 cells induced by HG stimulation

The CCK8 assay was conducted to identify the optimal PGE1 concentration and intervention time for subsequent cell experiments. HK-2 cells were treated with different concentrations of PGE1 $(0.1,1,10$, and $100 \mu \mathrm{M}$ ) in an HG environment (30 $\mathrm{mM}$ D-glucose). In addition, the MG group ( $24.5 \mathrm{mM}$ mannitol $+5.5 \mathrm{mM}$ D-glucose) and HG group (30 mM D-glucose) were used as the control groups. Six groups were cultured for 24, 48, and $72 \mathrm{~h}$. As shown in Fig. 4a, cell survival in the HG group was decreased compared with that in the MG group, but there was no significant difference at $48 \mathrm{~h}$. Compared to cell survival after treatment with 0.1 to $100 \mu \mathrm{M}$ PGE1, cell survival after treatment with $10 \mu \mathrm{M}$ PGE1 was higher, and the difference between the $10 \mu \mathrm{M}$ PGE1 group and the MG group was statistically significant $(P<0.05)$. Therefore, the optimal concentration and intervention time were $10 \mu \mathrm{M}$ and $48 \mathrm{~h}$, respectively.
Then, the cells were divided into three groups: the MG group, HG group, and HG + PGE1 group. The TUNEL assay indicated that the number of apoptotic cells was the highest in the HG group (white arrow, Fig. 4b). The rate of apoptosis in tubular cells in the HG group was $24.58 \% \pm 1.00 \%$, which was obviously increased compared with that in the MG group $(P<0.01)$. There was a reduction in the apoptosis rate in the PGE1 group $(5.27 \% \pm 1.70 \%)$ compared with that in the HG group, and the difference was statistically significant $(P<0.01$, Fig. $4 c)$.

Similarly, the cells were divided into three groups (the MG group, HG group, and HG + PGE1 group) for the flow cytometry assay. As shown in Fig. 4d, there was a statistically significant increase in cell apoptosis in the HG group compared with that in the control group $(P<0.05)$. Moreover, the apoptosis rate in the PGE1 group was significantly decreased compared with that in the HG group $(P<0.05)$. Taken together, our results suggest that PGE1 alleviates cell apoptosis in vitro.

PGE1 lessened the expression of Bim, Bax, caspase-3, and cleaved caspase- 3 in HK-2 cells induced by HG stimulation

We further explored the anti-apoptotic effect of PGE1 in vitro. Immunofluorescence staining showed that Bim expression increased after incubation with HG for $48 \mathrm{~h}$. PGE1 treatment obviously reduced Bim expression, which was consistent with our animal experiments (Fig. 5a).

Similarly, the expression levels of Bim, Bax, caspase- 3 and cleaved caspase- 3 were detected in cultured cell lines. Western blot and qRT-PCR results showed that Bim, Bax, caspase-3, and cleaved caspase-3 levels were reduced remarkably in the PGE1 group compared with those in the HG group (Fig. 5b-f). 
a
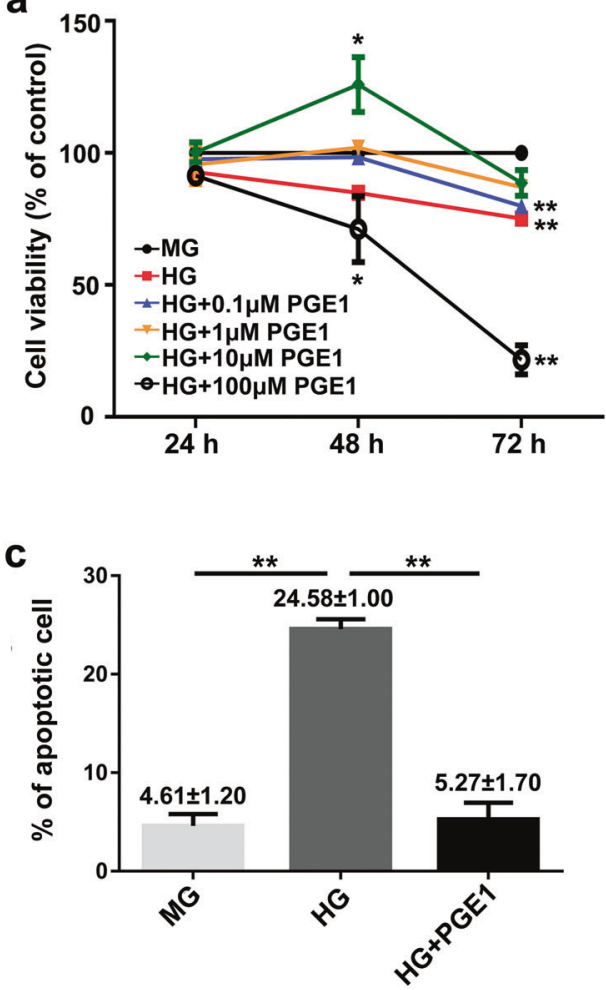

b
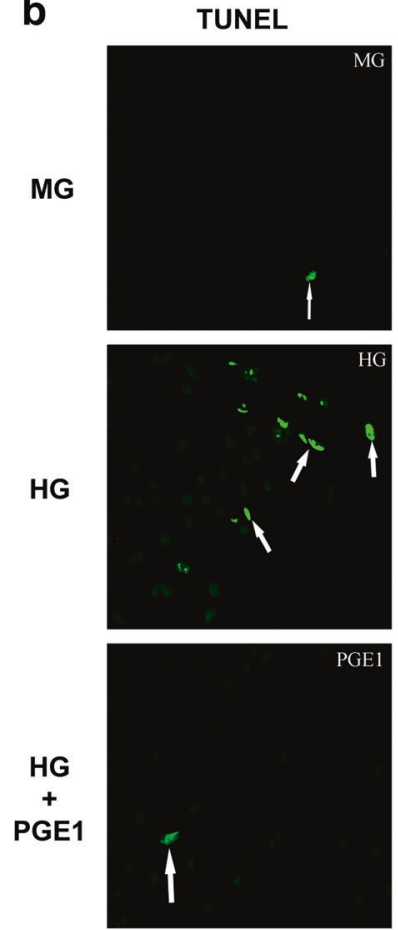

DAPI
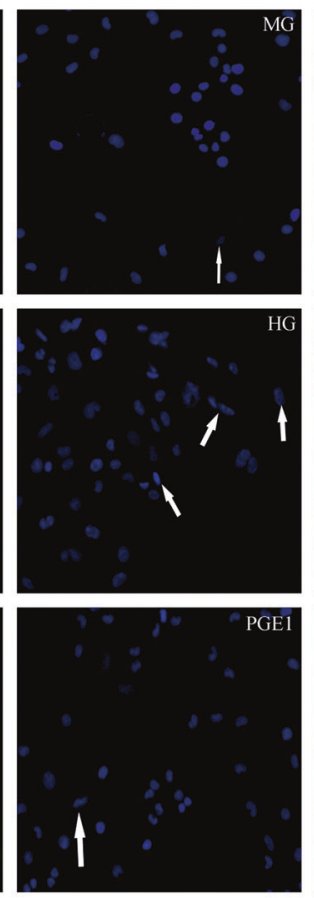
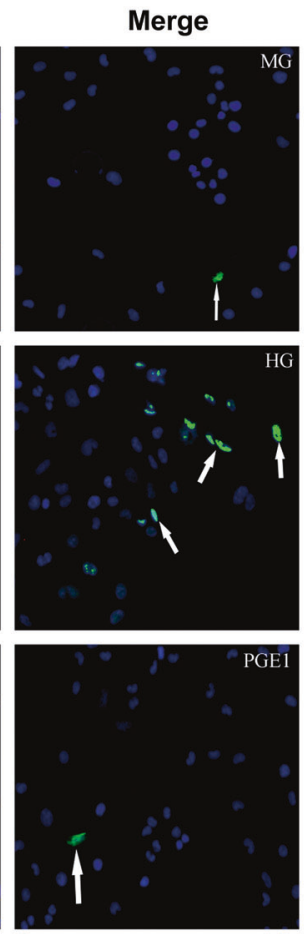

d
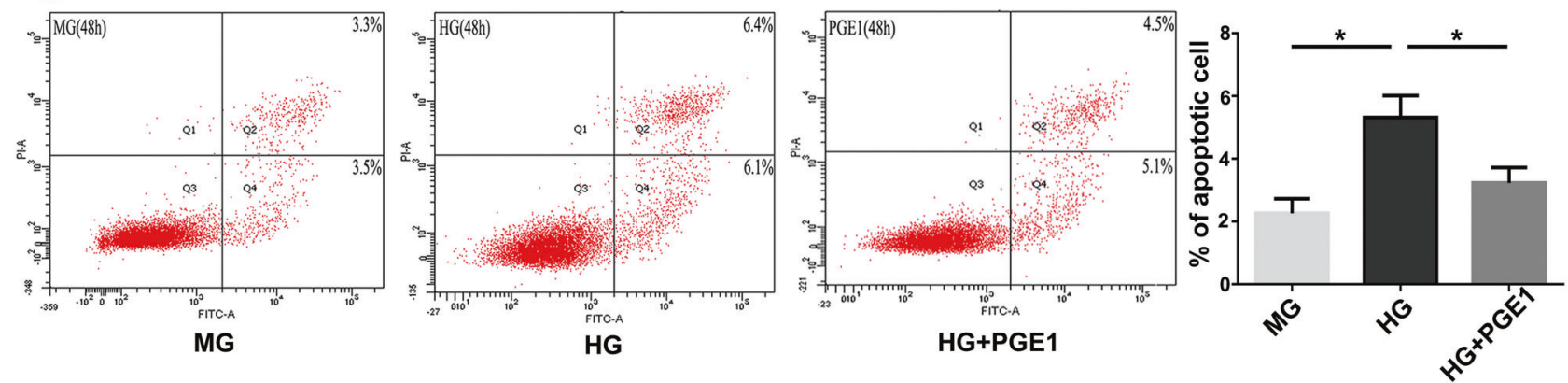

Fig. 4 PGE1 increased cell viability and reduced HG-induced apoptosis in HK-2 cells. a Cell viability was assessed by the CCK-8 assay after treatment with PGE1 at different concentrations for $24 \mathrm{~h}, 48 \mathrm{~h}$, and $72 \mathrm{~h}$ in HK-2 cells. The data represent the means \pm SEM. ${ }^{*} P<0.05$ versus the MG group. MG: mannitol $(24.5 \mathrm{mM})+$ glucose $(5.5 \mathrm{mM})$ group; HG: high glucose $(30 \mathrm{mM})$ group; PGE1: $0.1-100 \mu M$ prostaglandin E1. b, c TUNEL staining of HK-2 cells ( $\times 200$ magnification). The green fluorescence represents apoptotic cells, the blue fluorescence indicates nuclei, and the white arrows show apoptotic cells. The number of apoptotic cells and the total number of cells in 10 consecutive horizons were calculated. The rate of apoptosis was calculated by the following formula: the number of apoptotic cells in 10 consecutive horizons/the total number of cells $\times 100 \%$. The data represent the means \pm SEM. $* * P<0.01$ versus the HG group. $\mathbf{d}$ The apoptosis of HK-2 cells was detected by flow cytometry. The Q4 region represents early apoptotic cells, and the Q2 region represents terminal apoptotic and necrotic cells. The total apoptosis rate was calculated based on the Q2 + Q4 cell apoptosis ratio. The data represent the means \pm SEM. ${ }^{*} P<0.05$ versus the HG group

PGE1 inhibited the phosphorylation of JNK and Bim expression in HK-2 cells in HG

It has been postulated that JNK is an important regulator of apoptosis $[17,18]$. Hence, we investigated whether PGE1 affects JNK phosphorylation. As expected, PGE1 inhibited the phosphorylation of JNK while decreasing Bim expression. There was a significant reduction in the expression of $\mathrm{p}$-JNK and Bim in the HG + PGE1 group $(P<0.01, P<0.05$, respectively, Fig. 5d, e).

PGE1 blocked the activation of JNK/Bim in cultured HK-2 cells in HG

JNK and p-JNK expression was determined using Western blotting to verify whether JNK participates in the PGE1-mediated reduction in Bim in an HG environment. Intriguingly, we observed a positive correlation between the expression of JNK and Bim; the expression of $\mathrm{p}$-JNK increased with the prolongation of HG culture time $(1,6,12,24$, and $48 \mathrm{~h})$, and Bim expression subsequently increased. At $48 \mathrm{~h}$, the expression levels of $\mathrm{p}-\mathrm{JNK}$ and Bim were the highest (Fig. 6a, b). Therefore, we explored the regulatory relationship between JNK and Bim after $48 \mathrm{~h}$ of cell culture. The expression levels of $\mathrm{p}$-JNK and Bim were obviously higher than that in the HG group after incubation with the JNK agonist anisomycin (AM) in HG for $48 \mathrm{~h},(P<0.05, P<0.01$, respectively, Fig. $6 c$, d). Simultaneously, p-JNK and Bim expression levels were decreased significantly compared with those in the HG group after incubation with the JNK inhibitor SP600125 in HG $(P<0.05$, Fig. 6c, d). These results showed that the regulation of JNK by HG stimulation impacted Bim expression. Because there are many factors that affect Bim expression under $\mathrm{HG}$ conditions and to further define whether JNK is an upstream regulator of Bim, the 
Bim
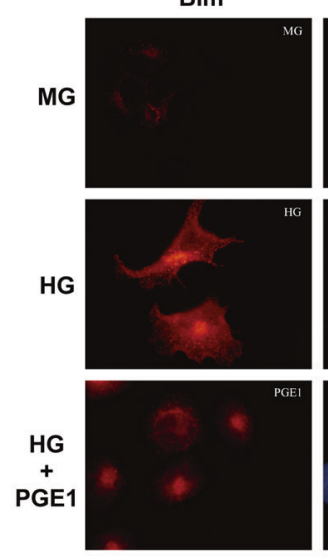

d $\mathrm{kDa}$
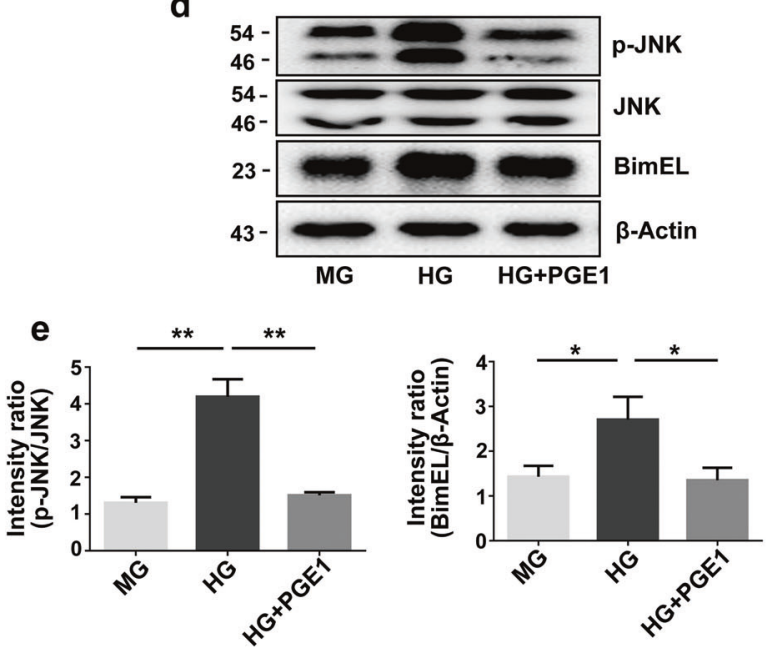

b
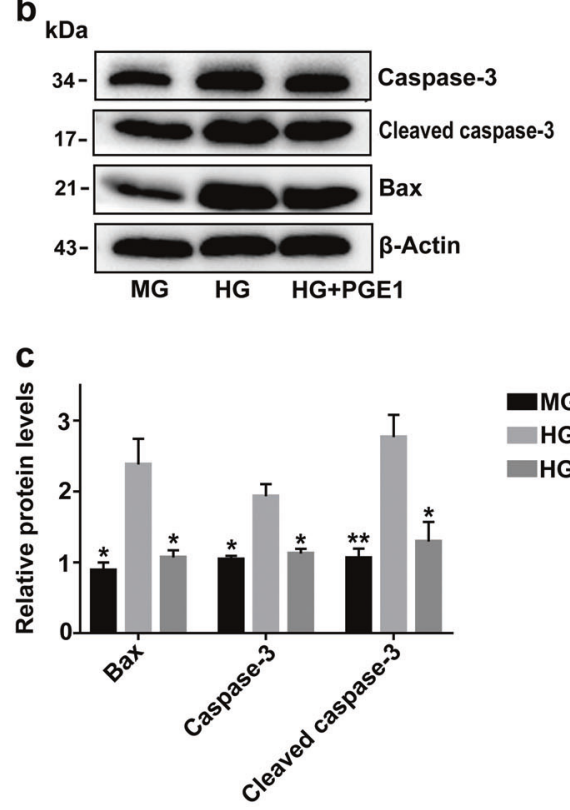

MG

HG

HG+PGE1

f

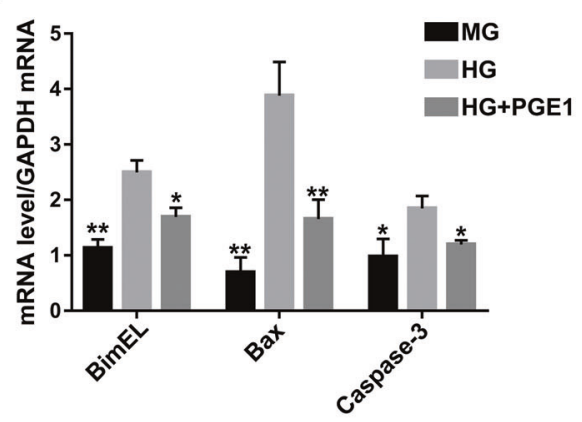

Fig. 5 PGE1 suppressed Bim, Bax, caspase-3, cleaved caspase-3 expression and phosphorylation of JNK in HK-2 cells. a Bim expression in HK-2 cells was detected by immunofluorescence (X600 magnification). The red fluorescence represents Bim expression, and blue fluorescence represents nuclei. b, c Western blot analysis of Bax, caspase-3 and cleaved caspase-3 expression in the MG, HG and HG + PGE1 groups. The data represent the means \pm SEM. ${ }^{*} P<0.05,{ }^{*} P<0.01$ versus HG group. $\mathbf{d}$, e Western blot analysis of Bim, $\mathrm{p}-\mathrm{JNK}$ and JNK expression in the MG, HG and HG + PGE1 groups. f qRT-PCR was performed to determine the mRNA levels of Bim, Bax, and caspase-3 in HK-2 cells. The data represent the means \pm SEM. ${ }^{*} P<0.05,{ }^{* *} P<0.01$ versus the HG group

regulation of JNK by MG alone was evaluated by detecting the expression of Bim after $48 \mathrm{~h}$. SP600125 and PGE1 had no effect on the expression of JNK under MG conditions (Fig. 6e, f). However, the expression of $\mathrm{p}$-JNK and Bim significantly increased upon incubation with AM compared with that in the MG group $(P<$ 0.01). SP600125 suppressed p-JNK expression after AM stimulation in MG $(P<0.05)$. Bim expression decreased synchronously $(P<$ 0.01). Along with the HG culture data, the results showed that Bim was mediated by JNK under both high and normal glucose conditions. Similarly, PGE1 also reduced the expression of p-JNK and Bim upon AM incubated in MG compared to that in the MG + AM group, as did SP600125 $(P<0.05, P<0.01$, respectively, Fig. $6 \mathrm{e}$, f). These data provided key evidence that the inhibition of JNK/ Bim signaling was indeed responsible for the alleviation of apoptosis by PGE1.

Moreover, we tested whether PGE1 regulates Bim through the other pathway. The HG + AM + PGE1 group was examined, and PGE1 partially ameliorated the expression of $\mathrm{p}-\mathrm{JNK}$ and Bim induced by AM compared with that in the HG + AM group $(P<$ 0.01 , Fig. $6 g$, h). However, although PGE1 decreased the expression of $\mathrm{p}-\mathrm{JNK}$ and $\mathrm{Bim}$ in the HG + AM + PGE1 group, the expression of $\mathrm{p}-\mathrm{JNK}$ and Bim were still higher than those in the HG + PGE1 group $(P<0.05$, Fig. $6 \mathrm{~g}, \mathrm{~h})$. Therefore, these results indicated that JNK/Bim signaling contributed to the inhibition of Bim induced by PGE1 treatment in HK-2 cells in HG.

\section{DISCUSSION}

The number of DM patients reached 451 million by the end of 2017, and it is estimated that the total number of DM patients will reach 693 million in 2045 [35]. As one of the most common chronic complications of diabetes, DKD has become one of the main causes of death from chronic kidney disease as well as the most common cause of end-stage renal failure in developed countries [36]. At present, strict control of blood glucose and blood pressure, especially the application of renin angiotensin inhibitor [37], cannot effectively control the occurrence and development of DKD. It has become urgent to clarify the pathogenesis of DKD and search for effective measures to prevent and delay DKD.

Previous studies have focused on glomerular lesions, but in recent years, a growing body of evidence has shown that renal tubular injury may be equally important [5-7], and apoptosis is more common in renal tubular lesions [38-40]. PGE1 is a powerful vasodilator involved in many physiological functions and has been corroborated as having an ameliorative effect on diabetic 
a

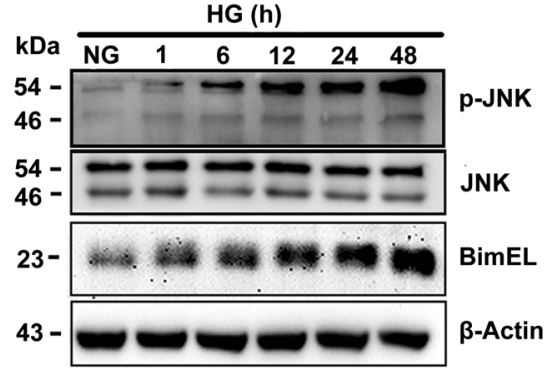

C

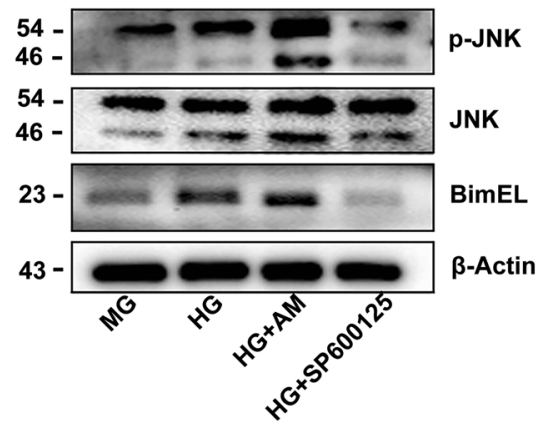

e

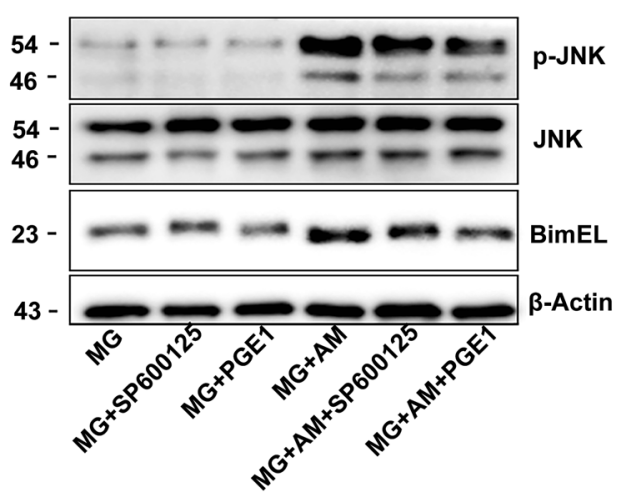

g

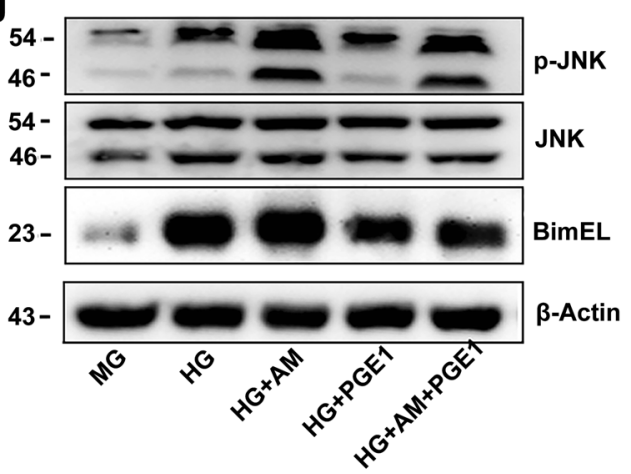

b
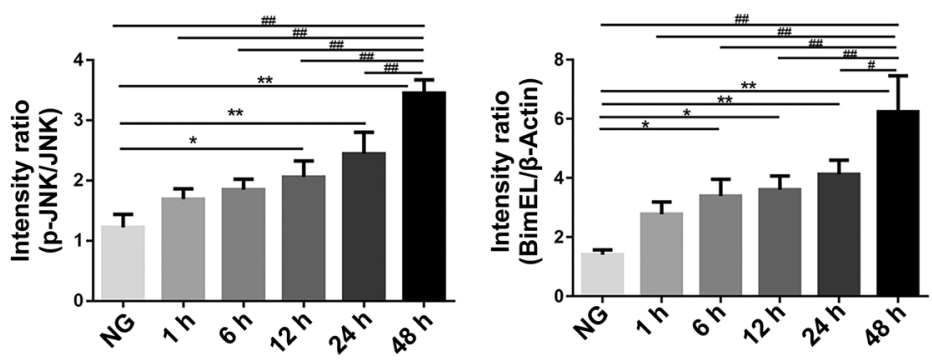

d
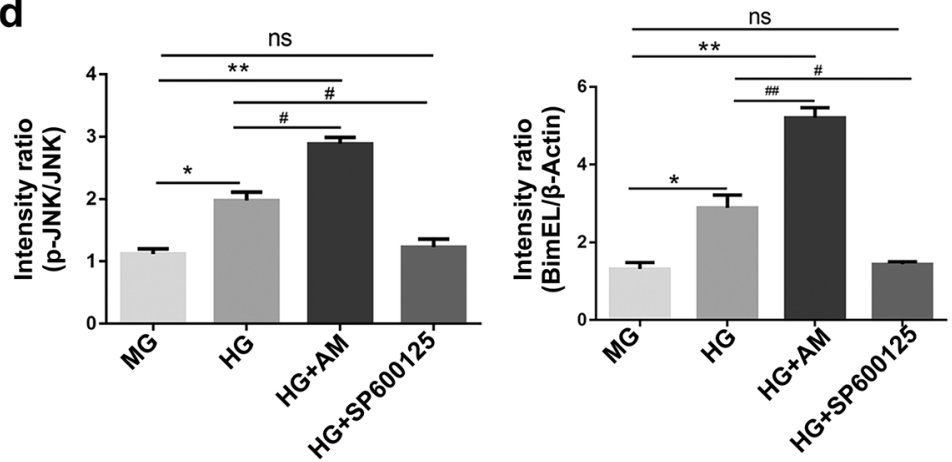

f
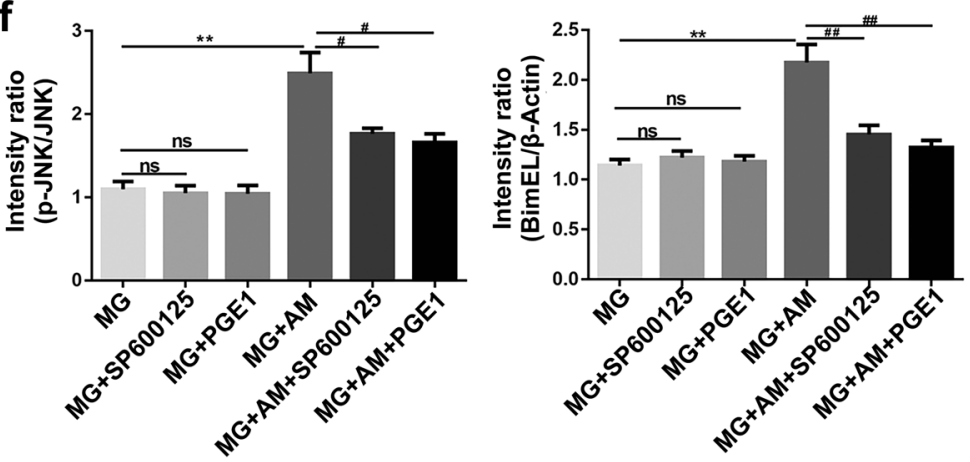

h
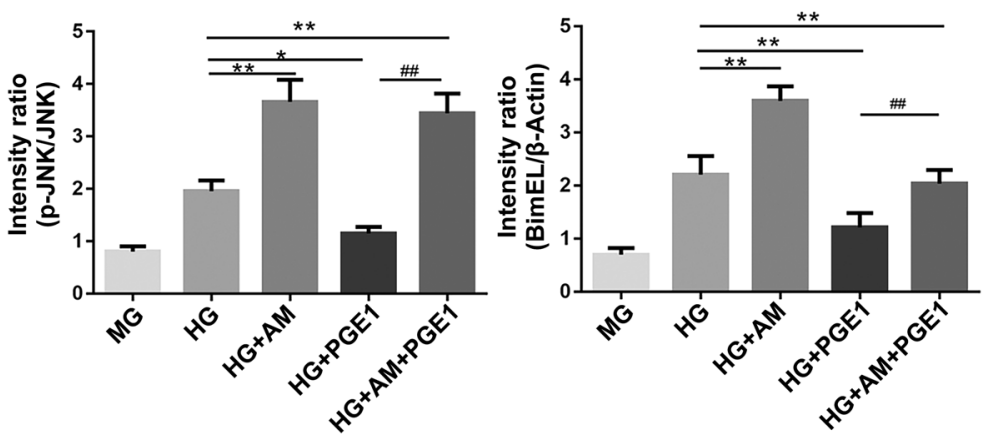

Fig. 6 PGE1 alleviated the activation of JNK/Bim induced by HG stimulation. $\mathbf{a}$, $\mathbf{b}$ Western blot analysis of $\mathrm{p}$-JNK, JNK and Bim expression after incubation with HG for different times $(1,6,12,24$, and $48 \mathrm{~h})$. The data represent the means $\pm \mathrm{SEM}$. ${ }^{*} P<0.05,{ }^{* *} P<0.01$ versus the NG group. ${ }^{\#} P<0.05,{ }^{\# \#} P<0.01$ versus the HG group at $48 \mathrm{~h}$. c, d Western blot analysis of p-JNK, JNK and Bim expression in the MG, HG, and HG + AM, $\mathrm{HG}+\mathrm{SP} 600125$ groups after $48 \mathrm{~h}$. The data represent the means \pm SEM. ${ }^{*} P<0.05,{ }^{* *} P<0.01$ versus the MG group. ${ }^{\#} P<0.05,{ }^{\# \#} P<0.01$ versus the HG group. e, $\mathbf{f}$ Western blot analysis of p-JNK, JNK and Bim expression in the MG, MG + SP600125, MG + PGE1, MG + AM, MG + AM + SP600125, and MG + AM + PGE1 groups. The data represent the means \pm SEM. ${ }^{* *} P<0.01$ versus the MG group. ${ }^{\#} P<0.05$, ${ }^{\# \#} P<0.01$ versus the MG + AM group. $\mathbf{g}, \mathbf{h}$ Western blot analysis of $\mathrm{p}-\mathrm{JNK}$, JNK, and Bim expression in the MG, HG, HG + AM, HG + PGE1, and HG + AM + PGE1 groups. The data represent the means \pm SEM. ${ }^{*} P<0.05,{ }^{* *} P<0.01$ versus the HG group. ${ }^{\# \#} P<0.01$ versus the HG + PGE1 group 
complications such as peripheral neuropathy [41]. A growing body of evidence has confirmed the apoptosis-inhibiting function of PGE1. Previous research has demonstrated that PGE1 reduces neuronal apoptosis induced by ischaemia/reperfusion [22]. Human umbilical vein endothelial cells are protected from $\mathrm{H}_{2} \mathrm{O}_{2}$ induced apoptosis by PGE1 in vivo [23]. In addition, PGE1 inhibits apoptosis via the downregulation of Bax expression and the upregulation of $\mathrm{BCl}-2$ expression in neonatal rat cardiomyocytes under hypoxic conditions [24]. Previously, we found that PGE1 alleviates urinary protein levels by inhibiting the apoptosis of renal tubular epithelial cells in DKD rats [25]. Notably, illuminating how PGE1 affects proximal renal tubular apoptosis might be helpful for identifying the pharmacological mechanism of PGE1. In the present study, we examined HG-induced toxicity in proximal renal tubular cells both in vivo and in vitro. The reversal of the apoptosis of proximal renal tubular cells and the alleviation of renal pathology, as determined by the TUNEL assay and immunohistochemistry, may indicate the cause of the reduction in proteinuria in DKD rats by PGE1 treatment.

The Bim protein has been found to play a vital role in the apoptotic process. Bim is part of the $\mathrm{BCl}-2$ family, which belongs to the proapoptotic gene family [16], and its activation leads to the activation of the mitochondrial apoptotic pathway. In our study, the protein expression of Bim, Bax, caspase-3 and cleaved caspase- 3 was increased in DKD rats, which is in accordance with other studies [42-44]. After $10 \mathrm{~d}$ of PGE1 treatment, the expression of these proteins was decreased. Additionally, immunofluorescence indicated that PGE1 reduced Bim expression in renal tubules as well.

Given that Bim is expressed mainly in proximal renal tubules [45], we further studied the HK-2 cell line to clarify the effects upstream of Bim. We found that PGE1 treatment not only reduced the expression levels of Bim, Bax, and cleaved caspase- 3 but also inhibited the phosphorylation of JNK in proximal renal tubular cells.

The course of apoptosis requires synergy between a variety of molecules, of which JNK is an important regulator. JNK is involved in many cell apoptotic processes, such as nervous system disease, type 1 diabetes, and tumor development and growth. In type 1 diabetes, the apoptosis of islet beta cells is associated with the activation of JNK [46]. In neuronal cells, JNK participates in Bimdependent apoptosis [19-21]. In addition, studies have confirmed that JNK is involved in kidney injury in diabetic rats and STZinduced DKD rats $[17,18]$. Therefore, we validated whether JNK is a key upstream regulator of Bim and whether JNK participates in PGE1-reduced Bim expression.

This study demonstrated that p-JNK and Bim protein levels were markedly elevated after incubation with HG over time, suggesting a positive correlation between JNK and Bim. After a JNK agonist and inhibitor were added, p-JNK and Bim expression was activated and obviously suppressed, establishing JNK as an upstream factor that regulates Bim-induced apoptosis. An important aspect of this study was the demonstration that JNK modulated Bim under both HG and normal glucose conditions, which indicates the direct regulation of JNK and the elimination of the effect of $\mathrm{HG}$ by Bim. Our results also indicated that JNK contributed to the PGE1-induced reduction in Bim. Activated $p$ JNK and Bim were more greatly reduced upon incubation with PGE1 and a JNK agonist than upon incubation with a JNK agonist alone. However, the expression of $p$-JNK and Bim were still higher than upon incubation with PGE1 alone, suggesting that PGE1 attenuated HG-induced Bim expression by inhibiting JNK.

In conclusion, our findings indicate that $\mathrm{HG}$ promotes the apoptosis of proximal renal tubular epithelial cells via the activation of JNK and the increased expression of the apoptosisrelated proteins Bim, Bax, caspase-3 and cleaved caspase- 3 . We also found that JNK/Bim signaling contributes to the apoptosis of renal tubular cells induced by HG. Additionally, PGE1 reduces the apoptosis of proximal renal tubular cells induced by HG stimulation and suppresses the activation of the JNK/Bim signaling pathway. Above all, our results support a potential therapeutic role of PGE1 in preventing DKD and the apoptosis of renal proximal tubules.

\section{ACKNOWLEDGEMENTS}

This work was funded by National Natural Science Foundation of China (81670757, 81570742); the Shandong Provincial Natural Science Foundation (ZR2016HQ26); and the Grant for the Development of Science and Technology of Ji-nan City (201602172).

\section{AUTHOR CONTRIBUTIONS}

$\mathrm{LL}$ and JJD conceived and designed the experiments and wrote the manuscript; $\mathrm{YHZ}$, YQZ, CCG, LKW, and YJC performed the experiments and analyzed the data.

\section{ADDITIONAL INFORMATION}

Competing interests: The authors declare no competing interests.

\section{REFERENCES}

1. Conserva F, Gesualdo L, Papale M. A systems biology overview on human diabetic nephropathy: from genetic susceptibility to post-transcriptional and posttranslational modifications. J Diabetes Res. 2016;2016:7934504.

2. Gnudi L. Cellular and molecular mechanisms of diabetic glomerulopathy. Nephrol Dial Transpl. 2012;27:2642-9.

3. Diez-Sampedro A, Lenz O, Fornoni A. Podocytopathy in diabetes: a metabolic and endocrine disorder. Am J Kidney Dis. 2011;58:637-46.

4. Suh $\mathrm{JH}$, Miner $\mathrm{JH}$. The glomerular basement membrane as a barrier to albumin. Nat Rev Nephrol. 2013;9:470-7.

5. Zhang XQ, Dong JJ, Cai T, Shen X, Zhou XJ, Liao L. High glucose induces apoptosis via upregulation of Bim expression in proximal tubule epithelial cells. Oncotarget. 2017;8:24119-29.

6. Hasegawa K, Wakino S, Simic P, Sakamaki Y, Minakuchi H, Fujimura K, et al. Renal tubular Sirt1 attenuates diabetic albuminuria by epigenetically suppressing Claudin-1 overexpression in podocytes. Nat Med. 2013;19:1496-504.

7. Xiao L, Zhu X, Yang S, Liu F, Zhou Z, Zhan M, et al. Rap1 ameliorates renal tubular injury in diabetic nephropathy. Diabetes. 2014;63:1366-80.

8. Magri CJ, Fava S. The role of tubular injury in diabetic nephropathy. Eur J Intern Med. 2009;20:551-5.

9. Kumar D, Robertson S, Burns KD. Evidence of apoptosis in human diabetic kidney. Mol Cell Biochem. 2004;259:67-70.

10. Lo CS, Shi Y, Chenier I, Ghosh A, Wu CH, Cailhier JF, et al. Heterogeneous nuclear ribonucleoprotein $F$ stimulates Sirtuin-1 gene expression and attenuates nephropathy progression in diabetic mice. Diabetes. 2017;66:1964-78.

11. Cai T, Wu XY, Zhang XQ, Shang HX, Zhang ZW, Liao L, et al. Calcium dobesilate prevents diabetic kidney disease by decreasing bim and inhibiting apoptosis of renal proximal tubular epithelial cells. DNA Cell Biol. 2017;36:249-55.

12. Gilbert RE. Proximal tubulopathy: prime mover and key therapeutic target in diabetic kidney disease. Diabetes. 2017;66:791-800.

13. Akiyama T, Tanaka S. Bim: guardian of tissue homeostasis and critical regulator of the immune system, tumorigenesis and bone biology. Arch Immunol Ther Exp. 2011;59:277-87.

14. Tennant BR, Vanderkruk B, Dhillon J, Dai D, Verchere CB, Hoffman BG. Myt3 suppression sensitizes islet cells to high glucose-induced cell death via Bim induction. Cell Death Dis. 2016;7:e2233.

15. Shin ES, Huang Q, Gurel Z, Palenski TL, Zaitoun I, Sorenson CM, et al. STAT1mediated Bim expression promotes the apoptosis of retinal pericytes under high glucose conditions. Cell Death Dis. 2014;5:e986.

16. Sionov RV, Vlahopoulos SA, Granot Z. Regulation of Bim in health and disease. Oncotarget. 2015;6:23058-134.

17. Han H, Cao A, Wang L, Guo H, Zang Y, Li Z, et al. Huangqi decoction ameliorates streptozotocin-induced rat diabetic nephropathy through antioxidant and regulation of the TGF-beta/MAPK/PPAR-gamma signaling. Cell Physiol Biochem. 2017;42:1934-44.

18. Pan $Y$, Zhang $X$, Wang $Y$, Cai L, Ren $L$, Tang $L$, et al. Targeting JNK by a new curcumin analog to inhibit NF-KB-mediated expression of cell adhesion molecules attenuates renal macrophage infiltration and injury in diabetic mice. PLoS One. 2013;8:e79084

19. Harris CA, Johnson EM Jr. BH3-only Bcl-2 family members are coordinately regulated by the JNK pathway and require Bax to induce apoptosis in neurons. J Biol Chem. 2001;276:37754-60 
20. Whitfield J, Neame SJ, Paquet L, Bernard O, Ham J. Dominant-negative c-Jun promotes neuronal survival by reducing BIM expression and inhibiting mitochondrial cytochrome $c$ release. Neuron. 2001;29:629-43.

21. Putcha GV, Moulder KL, Golden JP, Bouillet P, Adams JA, Strasser A, et al. Induction of BIM, a proapoptotic BH3-only BCL-2 family member, is critical for neuronal apoptosis. Neuron. 2001;29:615-28.

22. Kawamura T, Akira T, Watanabe M, Kagitani Y. Prostaglandin E1 prevents apoptotic cell death in superficial dorsal horn of rat spinal cord. Neuropharmacology. 1997;36:1023-30.

23. Fang WT, Li HJ, Zhou LS. Protective effects of prostaglandin E1 on human umbilical vein endothelial cell injury induced by hydrogen peroxide. Acta Pharmacol Sin. 2010;31:485-92.

24. Ma XQ, Fu RF, Feng GQ, Wang ZJ, Ma SG, Weng SA. Hypoxia-reoxygenationinduced apoptosis in cultured neonatal rat cardiomyocyets and the protective effect of prostaglandin E. Clin Exp Pharmacol Physiol. 2005; 32:1124-30.

25. Mou Y, Zhang Y, Guo C, Zhao J, Zhang Z, Zhou X, et al. Integrated treatment of prostaglandin $\mathrm{E} 1$ and angiotensin-converting enzyme inhibitor in diabetic kidney disease rats: possible role of antiapoptosis in renal tubular epithelial cells. DNA Cell Biol. 2018;37:133-41.

26. Zhao T, Zhang H, Zhao T, Zhang $X$, Lu J, Yin T, et al. Intrarenal metabolomics reveals the association of local organic toxins with the progression of diabetic kidney disease. J Pharm Biomed Anal. 2012;60:32-43.

27. Pari L, Sankaranarayanan C. Beneficial effects of thymoquinone on hepatic key enzymes in streptozotocin-nicotinamide induced diabetic rats. Life Sci. 2009;85:830-4.

28. Sutariya B, Saraf MB. isolated from fruits of Opuntia elatior Mill attenuates renal fibrosis in diabetic rats through regulating oxidative stress and TGF-beta pathway. J Ethnopharmacol. 2017;198:432-43.

29. Ling L, Zhang S, Ji Z, Huang H, Yao G, Wang M, et al. Therapeutic effects of lipoprostaglandin $\mathrm{E} 1$ on angiogenesis and neurogenesis after ischemic stroke in rats. Int J Neurosci. 2016;126:469-77.

30. Huang L, Haylor JL, Hau Z, Jones RA, Vickers ME, Wagner B, et al. Transglutaminase inhibition ameliorates experimental diabetic nephropathy. Kidney Int. 2009;76:383-94.

31. Meng XM, Huang XR, Xiao J, Chen HY, Zhong X, Chung AC, et al. Diverse roles of TGF-beta receptor II in renal fibrosis and inflammation in vivo and in vitro. J Pathol. 2012;227:175-88.

32. Fujii $N$, Matsuo $Y$, Matsunaga $T$, Endo $S$, Sakai $H$, Yamaguchi $M$, et al. Hypotonic stress-induced down-regulation of Claudin-1 and -2 mediated by dephosphorylation and clathrin-dependent endocytosis in renal tubular epithelial cells. J Biol Chem. 2016:291:24787-99.
33. Hirai $Y$, lyoda $M$, Shibata $T$, Kuno $Y$, Kawaguchi $M$, Hizawa $N$, et al. IL-17A stimulates granulocyte colony-stimulating factor production via ERK1/2 but not $\mathrm{p} 38$ or JNK in human renal proximal tubular epithelial cells. Am J Physiol Renal Physiol. 2012;302:F244-50.

34. Huang XR, Chung AC, Zhou L, Wang XJ, Lan HY. Latent TGF-beta1 protects against crescentic glomerulonephritis. J Am Soc Nephrol. 2008;19:233-42.

35. Cho NH, Shaw JE, Karuranga S, Huang Y, da Rocha Fernandes JD, Ohlrogge AW, et al. IDF diabetes atlas: global estimates of diabetes prevalence for 2017 and projections for 2045. Diabetes Res Clin Pract. 2018;138:271-81.

36. Subramanian S, Hirsch IB. Diabetic Kidney Disease: Is there a role for glycemic variability? Curr Diab Rep. 2018;18:13.

37. Abuissa $\mathrm{H}, \mathrm{O}^{\prime}$ Keefe J Jr. The role of renin-angiotensin-aldosterone system-based therapy in diabetes prevention and cardiovascular and renal protection. Diabetes Obes Metab. 2008;10:1157-66

38. Yuan XP, Liu LS, Chen CB, Zhou J, Zheng YT, Wang XP, et al. MicroRNA-423-5p facilitates hypoxia/reoxygenation-induced apoptosis in renal proximal tubular epithelial cells by targeting GSTM1 via endoplasmic reticulum stress. Oncotarget. 2017;8:82064-77.

39. Yuan J, Shen $Y$, Yang $X$, Xie $Y$, Lin $X$, Zeng W, et al. Thymosin beta4 alleviates renal fibrosis and tubular cell apoptosis through TGF-beta pathway inhibition in UUO rat models. BMC Nephrol. 2017;18:314.

40. Li $\mathrm{H}$, Zhu X, Zhang J, Shi J. MicroRNA-25 inhibits high glucose-induced apoptosis in renal tubular epithelial cells via PTEN/AKT pathway. Biomed Pharmacother. 2017;96:471-9.

41. Wu JD, Tao S, Jin X, Jiang LL, Shen Y, Luo Y, et al. PGE1 improves diabetic peripheral neuropathy in patients with type 2 diabetes. Prostaglandins Other Lipid Mediat. 2016;126:24-8.

42. Kato M, Yuan H, Xu ZG, Lanting L, Li SL, Wang M, et al. Role of the Akt/FoxO3a pathway in TGF-beta1-mediated mesangial cell dysfunction: a novel mechanism related to diabetic kidney disease. J Am Soc Nephrol. 2006;17:3325-35.

43. Chuang PY, Yu Q, Fang W, Uribarri J, He JC. Advanced glycation endproducts induce podocyte apoptosis by activation of the FOXO4 transcription factor. Kidney Int. 2007;72:965-76.

44. Xiao HT, Wen B, Ning ZW, Zhai LX, Liao CH, Lin CY, et al. Cyclocarya paliurus tea leaves enhances pancreatic beta cell preservation through inhibition of apoptosis. Sci Rep. 2017;7:9155.

45. O'Reilly LA, Cullen L, Visvader J, Lindeman GJ, Print C, Bath ML, et al. The proapoptotic BH3-only protein Bim is expressed in hematopoietic, epithelial, neuronal, and germ cells. Am J Pathol. 2000;157:449-61.

46. Major $\mathrm{CD}$, Wolf BA. Interleukin-1 beta stimulation of c-Jun $\mathrm{NH}_{2}$-terminal kinase activity in insulin-secreting cells: evidence for cytoplasmic restriction. Diabetes. 2001;50:2721-8. 